\title{
Characterization of the Caenorhabditis elegans Tc1 transposase in vivo and in vitro
}

\author{
Jan C. Vos, Henri G.A.M. van Luenen, and Ronald H.A. Plasterk ${ }^{1}$ \\ The Netherlands Cancer Institute, Division of Molecular Biology, 1066 CX Amsterdam, The Netherlands
}

\begin{abstract}
We have investigated the function of the Tc1A gene of the mobile element Tc1 of Caenorhabditis elegans. Tc1 is a member of a family of transposons found in several animal phyla, such as nematodes, insects, and vertebrates. Two lines of evidence show that Tc1A encodes the transposase of Tc1. First, forced expression of the Tc1A protein in transgenic nematodes results in an enhanced level of transposition of endogenous Te1 elements. Second, DNase I footprinting and gel retardation assays show that Tc1A binds specifically to the inverted repeats at the ends of the element and that the Tc1A recognition site is located between base pairs 5 and 26 from the ends of Tc1. Functional dissection of the transposase shows the presence of two distinct DNA-binding domains. A site-specific DNA-binding domain is contained within the amino-terminal 63 residues of Tc1A; this region shows sequence similarity to the prokaryotic IS30 transposase. A second, general DNA-binding domain is located between amino acids 71 and 207. Our results suggest that Tc1 is more similar to prokaryotic insertion elements than to eukaryotic transposons such as P elements in Drosophila or Ac and En-1 in plants.
\end{abstract}

[Key Words: C. elegans; transposase; Tcl; DNA binding]

Received February 11, 1993; revised version accepted April 21, 1993.

The Tc1 transposable element of Caenorhabditis elegans is the prototype of a family of transposons that are not only found in nematodes but also in arthropods and vertebrates (Harris et al. 1988; Henikoff and Plasterk 1988; Brezinsky et al. 1990; Heierhorst et al. 1992; Henikoff 1992; Prasad et al. 1991). The Tc1 transposon is $1611 \mathrm{bp}$ long, has 54-bp terminal inverted repeats, and contains one major open reading frame (ORF) (Rosenzweig et al. 1983a). As with most members of the family, Tc1 is always flanked by a TA target site (Rosenzweig et al. 1983b; Mori et al. 1988a). Although the terminal 4 nucleotides (CATG) are conserved among Tc1-like elements, the length and sequence of the inverted repeats are highly diverse (Henikoff 1992).

The Tc1 element is present in all C. elegans strains tested, but the number of copies (between 30 and 300) and the frequency of transposition are variable. Tc1 excision is detectable in somatic cells of the common laboratory strains N2 and Bergerac (Emmons and Yesner 1984). In contrast, germ-line Tc1 transposition is restricted to Bergerac animals. Genetic loci, called mutators, are essential for this germ-line activity /Collins et al. 1987; Mori et al. 1988b). The mobile nature of certain mutators themselves, together with the cosegregation of Tc1 elements with mutator activity, indicates that specific Tc1 elements may be the cause of germ-line transposition (Mori et al. 1988b; Mori 1989; R.H.A. Plasterk, unpubl.). This could be the consequence of either differ-

${ }^{1}$ Corresponding author. ences in primary sequence or, more likely, the flanking sequences that influence the tissue-specific expression of the gene contained in Tc1.

A general characteristic of autonomous transposable elements is the presence of one or more genes encoding proteins involved in the transposition reaction. For instance, $\mathrm{P}$ elements in Drosophila contain a gene that can encode either a transposase or a repressor of transposition. Both proteins share the same DNA-binding domain, which mediates binding to a 10-bp internal sequence of $\mathrm{P}$ elements adjacent to the 31-bp terminal inverted repeats (Kaufman et al. 1989; Misra and Rio 1990). Interestingly, the transposase-binding site is located 16 bp from the $5^{\prime}$ inverted repeat and $4 \mathrm{bp}$ from the $3^{\prime}$ inverted repeat. The transposases encoded by the plant transposons En-1 and Ac also do not bind to the inverted repeat sequences but, rather, recognize subterminal repeat motifs that are asymmetrically located within the left and right arm of the transposon (Gierl et al. 1988; Kunze and Starlinger 1989). In contrast, the transposases encoded by prokaryotic elements, such as IS 30 and IS903, recognize sequences within the inverted repeat of their transposable element (Stalder et al. 1990; Derbyshire and Grindley 1992). Mu contains a transposasebinding site at each end of the transposon, like IS30 and IS903, as well as additional binding sites that are asymmetrically spaced at the left and right arm of the transposon (Craigie et al. 1984).

The first complete sequence of a Tc1 element revealed the presence of an ORF capable of encoding 273 residues 
(Rosenzweig et al. 1983a). The hypothesis that the encoded polypeptide is a transposase was supported by the observation that the 273-amino-acid protein, named TcA, when produced in Escherichia coli, showed a strong affinity for DNA. No sequence specificity, however, was detected in a South-Western analysis (Schukkink and Plasterk 1990). Sequence analysis of more Tc1 elements and the sequence of a Tc1 homolog in Caenorhabditis briggsae, indicated that the gene could actually contain an additional exon, $5^{\prime}$ of the large exon identified previously (Schukkink and Plasterk 1990; Prasad et al. 1991). However, analysis of Tc1 mRNA did not result in the identification of a spliced transcript; the analysis was hindered by readthrough transcripts resulting from the many copies of Tc1 in the genome (R.H.A. Plasterk, unpubl.).

Here we report the identification of the intron-exon structure of the Tc1A gene by forced expression of this gene in transgenic animals and analysis of the induced mRNA and protein. Furthermore, we studied the effect of TclA expression on Tc1 transposition and identified two distinct DNA-binding domains within TclA.

\section{Results}

\section{Induced expression of the Tcl transposase}

Previous efforts to detect either mRNAs or proteins encoded by the Tc1 element of C. elegans failed to demonstrate directly the presence of the putative transposase (R.H.A. Plasterk, unpubl.). Therefore, we forced the expression of the genetic information of $\mathrm{Tc} 1$ in transgenic nematodes. A heat shock promoter plus untranslated leader sequence (Stringham et al. 1992) was fused to the start codon of the first exon of the Tc1 gene and cloned into the vector pPD26.50 (Fire et al. 1990), which contains an unc-54 polyadenylation signal (see Material and methods).
A transgenic N2 line, named NL224, containing the $h s p-T c 1 A$ fusion construct was established (see Materials and methods) and characterized. Northern analysis showed the appearance of an abundant RNA of $\sim 1.7 \mathrm{~kb}$ in response to a heat shock, which is consistent with proper initiation at the heat shock promoter and the use of the unc-54 polyadenylation signal (Fig. 1A, cf. lanes 1 and 2). The signal seen in both lanes near the origin of the gel resulted from residual genomic DNA. A polyclonal antiserum raised against the carboxyl terminus of TcA (Schukkink and Plasterk 1990) was used to analyze the induction of the putative transposase (Fig. 1B). A polypeptide of $\sim 40 \mathrm{kD}$, which we named TclA, was detectable after a heat shock of $2 \mathrm{hr}$ at $33^{\circ} \mathrm{C}$ in transgenic NL224 animals (Fig. 1B, lanes 7-10). Fractionation of protein extracts demonstrated that TclA is a nuclear protein (data not shown). The protein was not detected without a heat shock (lane 6), or in heat-shocked nontransgenic N2 animals (lanes 1-5).

The appearance of a polypeptide of $40 \mathrm{kD}$ indicated that the coding information for TclA is contained within two exons. To determine the intron-exon borders precisely, an analysis of the mRNA induced with heat shock was performed by generating a cDNA that was subsequently amplified by PCR. Sequence analysis of the PCR product revealed that an intron of 41 nucleotides is removed from the pre-mRNA (Fig. 1C). This demonstrates that the $3^{\prime}$ splice site was predicted correctly but that the actual intron size is 41 nucleotides instead of the predicted 65 nucleotides (Schukkink and Plasterk 1990|. Taken together, these results show that Tc1 encodes a protein of 343 amino acids, which is synthesized in the transgenic NL224 line upon induction.

\section{Increase of Tc1 transposition on expression of Tc1A}

The effect of induction of TclA on the frequency of transposition of resident Tc1 elements was tested by
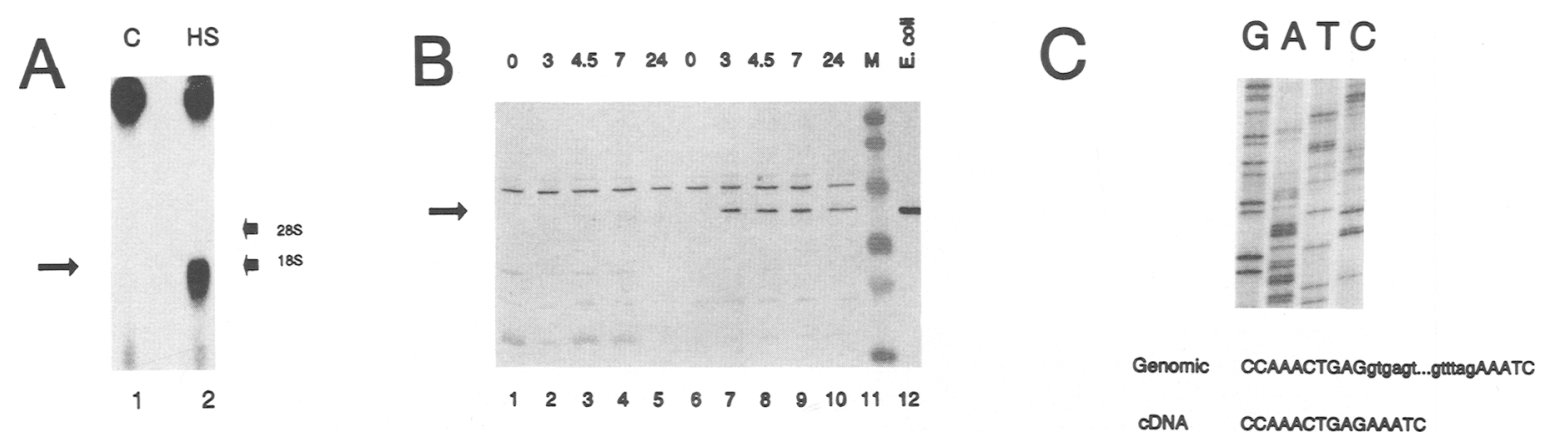

Figure 1. Inducible expression of Tc1A in transgenic line NL224. (A) Two micrograms of total RNA from line NL224 extracted before (C) or after (HS) a 3-hr heat shock was analyzed on a Northern blot using Tc1 DNA as probe. The position of the induced Tc1A mRNA is indicated with an arrow, as well as the positions of $18 \mathrm{~S}$ and $28 \mathrm{~S}$ RNAs. (B) Western blot analysis of N2 (lanes 1-5) and NL224 (lanes 6-10) using a Tc1A-specific polyclonal antiserum. From $t=0 \mathrm{hr}$, animals were heat shocked for $2 \mathrm{hr}$ and subsequently returned to $18^{\circ} \mathrm{C}$. Protein extracts were prepared at the times indicated $(\mathrm{hr})$ and separated on a $10 \%$ SDS-polyacrylamide gel. The position of Tcl A is indicated with an arrow. Lane $M$ contains prestained molecular mass markers of 106, 80, 49.5, 32.5, 27.5, and 18.5 kD. Lane 12 contains Tc1A produced in E. coli. $(C)$ Sequence analysis of Tc1A cDNA. A cDNA was made of heat shock-induced Tc1A mRNA, amplified by PCR, and subsequently sequenced. The exon sequence is given in uppercase letters, and the intron sequence is shown in lowercase letters. 
monitoring Tc1 insertions into the gpa-2 gene /Fino Silva and Plasterk 1990) using the specificity and sensitivity of PCR. This method allowed us to detect somatic transposition events. We did not detect germ-line transposition, which was not unexpected, because the $h s p-16$ promoter is only active in somatic cells (Stringham et al. 1992). Two rounds of PCR were performed with gpa-2and Tc1-specific primers. A PCR product was expected only when $T c 1$ had integrated in the proper orientation in the vicinity of the gpa-2 gene. The frequency of Tc1 transposition was measured as the number of PCR products per mass of DNA. Genomic DNA was prepared from both heat-shocked and non-heat-shocked N2 and transgenic NL224 worms. In 20 reactions, each containing $30 \mathrm{ng}$ of DNA derived from the NL224 animals in which Tc1A was induced, we found $20 \mathrm{Tc} 1$ insertions in gpa-2 (Fig. 2B). The size distribution of the PCR products indicates that different, independent transposition events are detected. In contrast, 20 reactions with $100 \mathrm{ng}$ of DNA derived from uninduced NL224 animals resulted in only three insertions (Fig. 2A). To exclude the possibility that the increase in transposition is the result of the heat shock itself, we performed a similar experiment with DNA prepared from N2 animals. Three insertion events were obtained in 20 PCRs with $100 \mathrm{ng}$ of DNA each prepared from non-heat-shocked N2 animals (Fig. $2 \mathrm{Cl}$, whereas the same amount of DNA from the heatshocked N2 animals yielded five insertions (Fig. 2D). This demonstrates that a moderate level of somatic transposition occurs in wild-type N2 animals, a process not influenced significantly by a heat shock treatment. Therefore, the $\sim 18$-fold increase in transposition in heatshocked NL224 animals is attributable to expression of TclA. The 18-fold increase is an underestimation of the effect of TclA expression, because the transgene is neither stably maintained within the nematode population nor within all cells of a transgenic animal, and because the heat shock promoter is not expressed in all somatic cells of C. elegans (Stringham et al. 1992). It is important to note that the expression of TclA did not result in detectable levels of transposition of a Tc1-related element, Tc3 (Collins et al. 1989), indicating that the activity of TclA is transposon specific (data not shown).

Several Tc1 insertions in gpa-2 were sequenced and found to be genuine transposition events as judged by the presence of the complete inverted repeat and the integration into a TA target sequence (data not shown). From the increase in Tc1 insertions after induction of Tc1A, we conclude that Tc1A is a limiting factor in the transposition reaction in vivo.

\section{Site-specific binding of Tc1A to the inverted repeat of $T c 1$}

One of the activities expected of Tc1 transposase is binding to a DNA sequence within the Tc1 element. We prepared cytoplasmic and nuclear extracts of the transgenic NL224 line and used those extracts to probe for sequence-specific binding to Tc1 fragments. The result of a gel-retardation assay is shown in Figure 3, in which the labeled DNA fragment contains the 26 terminal nucleotides of Tc1 as well as the flanking TA target dinucleotide. A band shift was observed using the nuclear extract prepared from NL224 animals with induced TclA expression (lane 4) and not with the nuclear extract from uninduced animals (lane 2). Cytoplasmic extracts (lanes 1,3) yielded no specific complex. The band shift obtained was the result of sequence-specific binding, because it was competed with an excess of unlabeled Tc1 oligonucleotide (lane 5) but not with an unrelated oligonucleotide (lane 6). These results suggest strongly that TclA has the ability to bind to the inverted repeat of Tc1 but do not exclude formally the possibility that
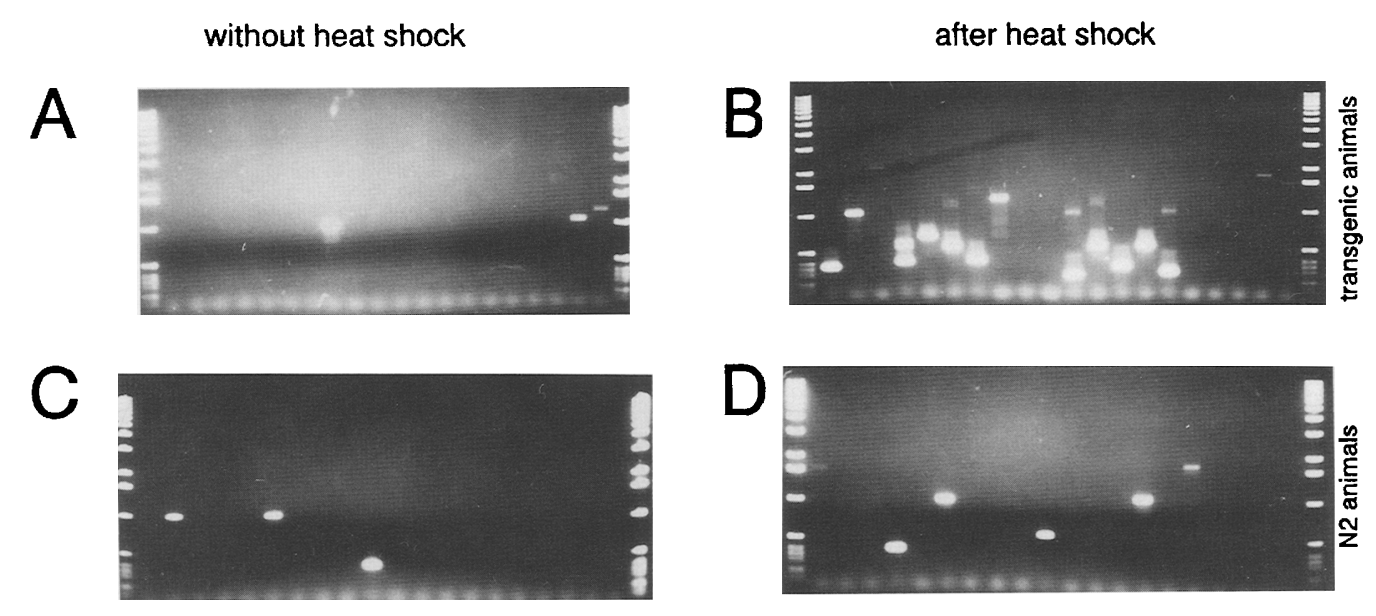

Figure 2. Effect of TclA expression on the frequency of Tc1 insertions in gpa-2. DNA was prepared from N2 $(C, D)$ and NL224 $(A, B)$ animals that had either received no heat shock $(A, C)$ or a 2 -hr heat shock followed by $18 \mathrm{hr}$ of recovery at $18^{\circ} \mathrm{C}(B, D)$. Tc1 insertions in gpa-2 were detected by PCR using Tc1- and gpa-2-specific primers. The products of 20 reactions using $30 \mathrm{ng}$ of DNA $(B)$ or $100 \mathrm{ng}$ of DNA $(A, C, D)$ were separated on a $1 \%$ agarose gel and stained with ethidium bromide. A 1-kb DNA ladder (BRL) was run in parallel as marker. 


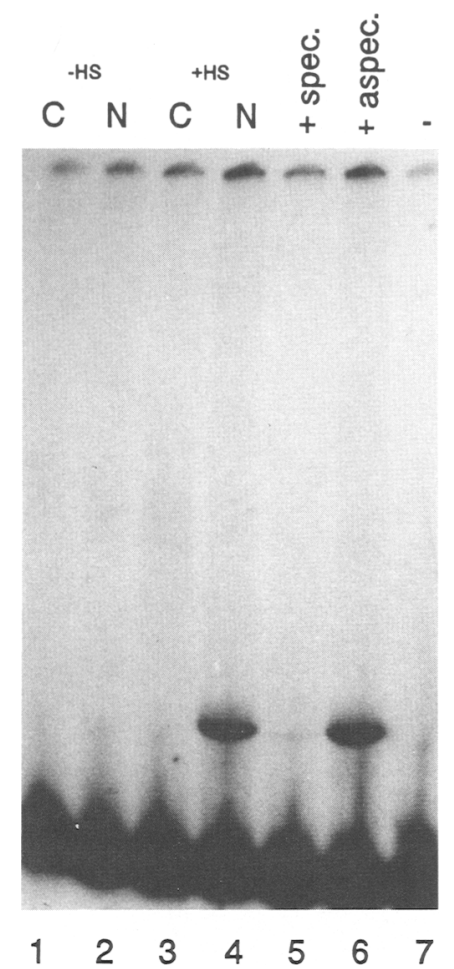

Figure 3. Tc1 ends specific DNA-binding activity in C. elegans extracts. A $36-\mathrm{bp}$ probe containing the 26 terminal bp of Tc1 flanked by the TA target sequence was mixed under standard conditions with $2 \mu$ l of either cytoplasmic $(\mathrm{C})$ or nuclear $(\mathrm{N})$ extract prepared from NL224 animals that had received either no heat shock (lanes 1,2) or a 2.5 -hr heat shock (lanes 3-6). In lane 7, no extract was added to the reaction. Ten picomoles of specific (lane 5) or nonspecific (lane 6) 28-mers were included in the binding assay. Products were separated on a native $5 \%$ polyacrylamide gel.

TclA expression induces or activates another protein that binds Tc1 ends.

To study the DNA-binding potential of TclA in more detail, we expressed the protein under the control of the T7 promoter in E. coli. The intron sequence was removed from the Tc1A gene (see Materials and methods), and the ORF was cloned into the vector pET3c (Rosenberg et al. 1987). Upon induction of T7 RNA polymerase, a prominent protein was synthesized, which comigrated with the $40-\mathrm{kD} \mathrm{Tcl} A$ protein produced in transgenic NL224 animals as shown in the Western blot analysis of Figure 1B (lane 12).

Bacterial lysates were made and analyzed in a bandshift assay. Two retarded complexes specific for Tc1 ends were obtained in the extract with the TclA expression vector (Fig. 4, lane 3) and were absent from the pET3c extract (lane 2). Surprisingly, neither of these complexes has the same mobility as the complex found with the nuclear NL224 extract (lane 1). Upon purification of the polypeptide responsible for the major shift, we obtained a fraction that did not contain the $40-\mathrm{kD}$ TclA protein but which did contain a polypeptide of $\sim 18$
$\mathrm{kD}$ with reactivity against an antiserum that recognizes an epitope between amino acids 71 and 153 of TclA. The polypeptide was not recognized by the antiserum against the carboxyl terminus of TclA (data not shown). Therefore, the complex resulted from binding of a TclA derivative, which was possibly the consequence of proteolysis of full-length TclA or of a premature translational stop. Binding of the full-length TclA protein resulted in the retarded complex near the origin of the gel. This complex is far less abundant, although full-length Tc1A is expressed at a much higher level than the TclA derivative. Thus, full-length TclA appears to have a low specific DNA-binding activity.

\section{Mapping of the site-specific DNA-binding domain of $\operatorname{Tc} 1 A$}

To map the domain of TclA that is responsible for binding to the inverted repeat of Tc1, we made carboxy-terminal deletion mutants and expressed them in E. coli (see Materials and methods). The expression of all of the deletion mutants was verified by SDS-PAGE /data not shown) and site-specific DNA binding was tested in a
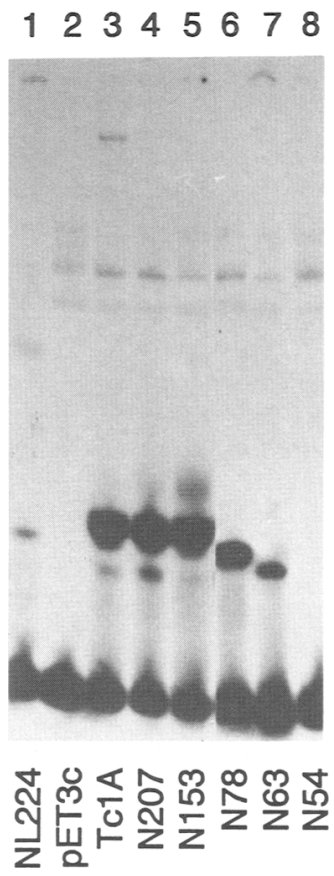

Figure 4. Specific DNA binding by recombinant TclA and carboxy-terminal deletion mutants. E. coli strain BL21 pLysS was transformed with pET3c-derived vectors for the expression of Tc1A as well as carboxy-terminal deletions thereof. Bacterial lysates were prepared from induced cultures and mixed under standard conditions with a 36-bp probe containing the ends of Tc1. The number of amino-terminal triplets of the Tc1A gene preserved in the expression vector are indicated at the bottom. Lane 2 represents the bacterial lysate obtained with the empty vector $\mathrm{pET} 3 \mathrm{c}$. In lane 1, $2 \mu \mathrm{l}$ of a nuclear extract prepared from heat-shock treated NL224 animals was used. Reaction products were separated on a native $5 \%$ polyacrylamide gel. 
gel-retardation assay with the terminal sequence of Tc1 as probe (Fig. 4). Because the major shift observed with the $\mathrm{Tcl} A$ expression plasmid is presumably caused by a smaller polypeptide, it is possible that deletions of carboxy-terminal sequences do not abolish the appearance of the major retarded complex. Extracts containing deletion mutants N207 (lane 4) and N153 (lane 5) showed the same prominent complex as the extracts with full-length TclA (lane 3). Thus, the genetic information required for the generation of the major retardation complex (lanes 3-5) is contained within the first 153 triplets of the Tc1A gene. Complexes corresponding to either the full-length N207 or N153 mutant proteins were not detected. Mutant N78 (lane 6) resulted in a retarded complex with a higher mobility than the one generated by the TclA derivative, suggesting that the deletion was beyond the position of the premature translational stop or the proteolytic cleavage. Mutant N63 (lane 7) gave rise to a complex with the highest mobility. The deletion mutant N54 appeared to be unable to bind to the terminal Tc1 sequence (lane 8). Apparently, the amino-terminal 63 amino acids of TclA are necessary and sufficient for sequence-specific binding to the inverted repeat of Tc1.

\section{DNase I footprinting analysis}

The site-specific binding of the TclA derivative was analyzed further in a DNase I footprint assay (Fig. 5A). The top strand was protected against DNase I attack by the TclA derivative from position 3 to 29 with respect to the end of the element. A slightly shifted protected region was obtained on the bottom strand-between positions 6 and 31. A schematic representation of the DNase I-protected region within the inverted repeat of $\mathrm{Tc} 1$ is given in Figure 5B. The footprint data are consistent with the binding specificity seen in the gel-retardation assays.

The DNase I footprint results suggest that the conserved terminal TACAGT sequence at the end of the transposon is not part of the transposase recognition sequence. To investigate this, we tested DNA binding of the TclA derivative to transposon ends with a mutated TACAGT sequence in a gel-retardation assay (Fig. 6). The TclA derivative bound with equal affinity to two different mutated sequences (lanes 3,4 ) and to the fragment with the authentic Tc1 sequence (lane 1), whereas an unrelated sequence was not recognized (lane 2). Therefore, the terminal TACAGT sequence is not essential for recognition of the ends of $\mathrm{Tc} 1$ by the Tc1A derivative.

\section{Mapping of a nonspecific, second DNA-binding domain}

A general DNA-binding domain has been defined for the Tc1A amino acids 71-235 (Schukkink and Plasterk 1990). This domain does not coincide with the minimal DNA-binding domain required for site-specific binding to the Tc1 inverted repeat defined above. We investigated the nonspecific DNA-binding domain further by analyzing the carboxy-terminal deletions in a South-

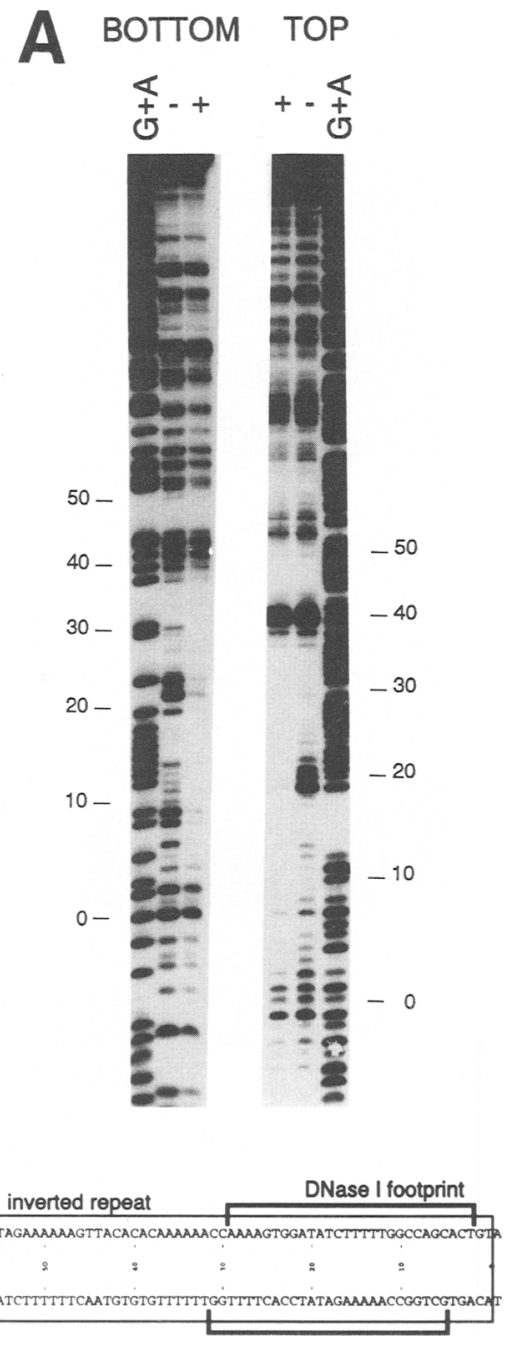

Figure 5. DNase I footprinting of transposase-DNA complexes. $|A|$ The footprint probe was a 274-bp EcoRI-HindIII fragment, which contains the terminal $225 \mathrm{bp}$ of the right end of Tc1, labeled at the HindIII site on either the top or bottom strand. DNase I footprints were obtained in the absence (lanes marked - ) or presence $(+)$ of $1 \mu$ of the partially purified TclAderivative. $G+A$ lanes are Maxam and Gilbert sequencing reactions on the same DNA. The numbering corresponds to the position relative to the end of the Tc1 element (see $B) .(B)$ Schematic representation of the footprint data. The inverted repeat is indicated by a box, and the protected region is marked by bars.

Western analysis using a labeled double-stranded random DNA sequence as probe (Fig. 7). DNA binding was observed with the full-length TclA protein (lane 1), as well as with mutant N207 (lane 2). On a long exposure, some DNA binding was still detectable with mutant N153 (lane 3), whereas no DNA binding was observed for any of the other mutants (lanes 4-7). Notably, the carboxy-terminal deletions that still contain the specific DNA-binding activity are negative for general DNA binding in this assay. Combining these data together with those of Schukkink and Plasterk (1990), we conclude that the nonspecific DNA-binding domain is be- 

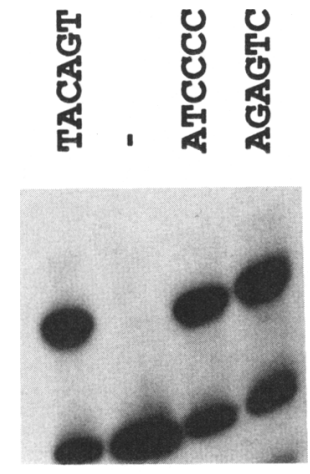

$\begin{array}{llll}1 & 2 & 3 & 4\end{array}$

Figure 6. Gel retardation analysis of the effect of the outer base pairs on binding of Tc1 transposase. Probes of $79 \mathrm{bp}$ were made that contained either no similarity to Tc1 ends (lane 2) or contained the Tc1 inverted repeat sequences between 5 and 26, flanked by the conserved TACAGT sequence (lane 1) or the mutated sequences ATCCCC (lane 3) or AGAGTC (lane 4). Probes were mixed with $0.05 \mu$ l of the partially purified Tc1A derivative, and reaction products were analyzed on a $4 \%$ polyacrylamide gel.

tween amino acid residues 71 and 207, whereas the sitespecific DNA-binding domain is within the first 63 residues.

\section{Similarity of the amino terminus of Tc1A withIS30 transposase}

In previous computer searches for homology between Tc1A and other proteins, the amino-terminal part of the

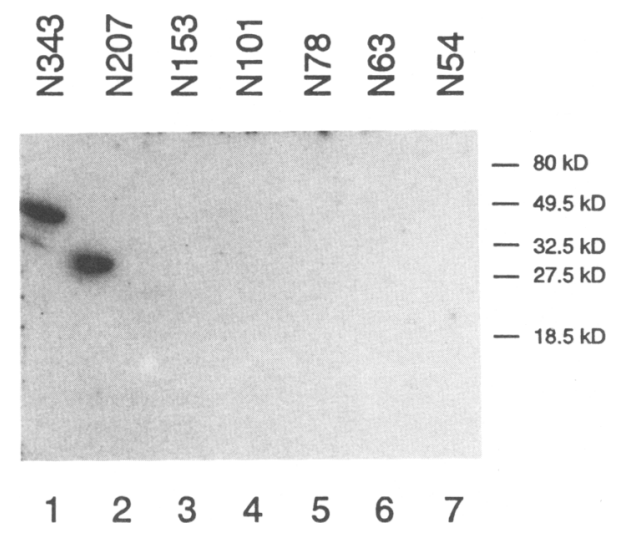

Figure 7. South-Western analysis of the general DNA-binding capacity of TclA and carboxy-terminal deletion mutants thereof. Total bacterial lysates $(10 \mu \mathrm{l})$ from induced BL21 pLysS carrying the expression vectors for TclA and various deletion derivatives were separated on a $12.5 \%$ SDS-polyacrylamide gel and transferred to nitrocellulose. The blot was probed with radiolabeled nonspecific DNA for $2 \mathrm{hr}$. The lanes are marked at the top with the number of amino-terminal codons of the Tc1A gene present in the expression vector. The positions and molecular masses of the marker proteins are shown at right. protein has been ignored because the $T c 1 A$ gene was considered to consist of only one exon. Once we mapped the site-specific DNA-binding domain to the amino terminus of TclA, we repeated the homology search using the BLAST program (Altschul et al. 1990) for the first 71 amino acids of TclA. The search revealed one striking homology (maximal segment pair score of 51) with IS30 transposase (Fig. 8) within an amino-terminal fragment of the transposase that is sufficient for site-specific binding to the terminal repeats of IS30 (Stalder et al. 1990). It requires further experimentation to verify the significance of the observed similarity.

\section{Discussion}

To test the hypothesis that the Tc1A gene of the transposable element $T c 1$ of $C$. elegans encodes a transposase, we generated a transgenic line in which the $T c 1 A$ gene is under the control of a heat shock promoter. Analysis of the mRNA revealed the intron-exon structure of the Tc1A gene; the actual size of the intron is smaller than initially predicted from DNA sequence data (Schukkink and Plasterk 1990). Overexpression of the $40-\mathrm{kD}$ TclA protein in C. elegans resulted in an enhanced level of transposition of resident Tc1 elements. This demonstrates that Tc1, like all autonomous mobile elements, contains a transposase gene and that its product is a limiting factor for transposition. The transposase is specific for Tc1 transposition, as overexpression did not result in transposition of Tc3, a Tc1-like transposable element in C. elegans. The transgenic NL224 line in which Tc1A expression is under the control of a somatic promoter can be characterized as an artificial somatic mutator. Likewise, it should be possible to establish an artificial germ-line mutator by fusion of the Tc1A gene to germ line-specific regulatory sequences. The natural germ-line mut-5 mutator activity is thought to be the result of enhanced expression of the Tc1A gene of a particular Tc1 element that has inserted in the neighborhood of a germline enhancer (Mori et al. 1988b).

We show site-specific binding of TclA to the inverted repeat of the transposable element. The primary recognition sequence is within base pairs 5 and 26 of the inverted repeat; substitution of the conserved terminal sequence TACAGT has no effect on binding of Tc1A. It has been suggested for prokaryotic transposons, such as Tn10 and IS903, that the outer base pairs are not important for initial transposase binding, but are involved in a later step in the transposition reaction (Huisman et al. 1989; Derbyshire and Grindley 1992). Currently, we are

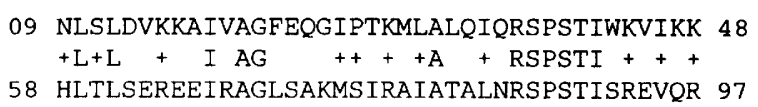

Figure 8. Homology between the DNA-binding domain of TclA and IS30 transposase. The similarity $|+|$ and identity (uppercase letters) are indicated between amino acid residues 9 and 48 of TclA (top) and residues 58 and 97 of IS30 transposase (bottom). 
investigating the consequence of mutations within the terminal nucleotides of Tc1 on transposition. The observation that TclA binds to the inverted repeat is more reminiscent of prokaryotic transposases than of eukaryotic transposases such as $\mathrm{P}$ transposase in Drosophila (Kaufman et al. 1989) or TnpA of En-1 (Gierl et al. 1988) and ORFa of Ac (Kunze and Starlinger 1989) in Zea mays. These transposases do not bind to inverted repeat sequences but to subterminal control regions that are asymmetrically spaced with respect to the transposon ends. The similarity of the DNA-binding domain of TclA with IS 30 transposase, which binds to the inverted repeat of IS30, is also suggestive of a closer relationship between the Tc1 family of transposons and prokaryotic insertion elements than between Tc1 and the abovementioned transposons characterized in plants and in Drosophila. Furthermore, sequence similarities have been found between the central part of TclA /triplets 103-285) and prokaryotic insertion elements (Henikoff 1992).

The proposed mechanism of Tc1 transposition is the excision of the element by double-strand cleavage and reintegration of the linear intermediate into a novel target site (Plasterk 1991). TclA is the most likely candidate for the execution of the double-strand break, because it binds to the Tc1 ends close to the cleavage sites. However, DNase I footprinting only shows protection by a TclA derivative of the DNA region from base pairs 3 to 31. It is possible that full-length Tc1A makes contacts with the terminal nucleotides, but, alternatively, contacts with the very ends of the transposon are only made after the formation of a synapse between the two Tc1 ends. This has been shown for $\mathrm{Mu}$, where the cleavage sites become protected from nuclease digestion after formation of the type 1 transpososome (Lavoie et al. 1991; Mizuuchi et al. 1991). The nonspecific DNA-binding domain of Tc1A, which we find to be distinct from its site-specific recognition domain, might be responsible for interactions with the target DNA.

We observed that a recombinant amino-terminal derivative of Tc1A binds Tc1 ends efficiently, whereas the full-length transposase has a very low affinity for Tc1 ends. The results obtained with the nuclear extract of the transgenic NL224 line point to a similar conclusion. They suggest the presence in the transgenic nematode of a relatively rare polypeptide, comparable in size to the recombinant TclA derivative, with affinity for Tc1 ends. We have not yet identified the polypeptide responsible. Possibly, an internal region of the Tc1A protein influences the DNA-binding potential of the amino-terminal domain. The structural basis of this theory is not yet understood, but there are precedents for cryptic DNAbinding domains. Recently, it has been reported that amino-terminal amino acids of the prokaryotic $\sigma^{70}$ transcription factor inhibit binding to promoter DNA (Dombroski et al. 1992). Also, the carboxyl terminus of human p53 has been shown to regulate DNA binding (Hupp et al. 1992).

Synthesis of a truncated version of a transposase that plays a role in the regulation of transposition is known for the P element (Misra and Rio 1990), Tn5 (Johnson et al. 1982; Isberg et al. 1982), and IS1 (Escoubas et al. 1991). These transposons regulate the level of transposition by controlling the relative abundance of a smaller version of the full-length transposase. These smaller versions still contain the domain for binding to specific sites on the transposon and act as inhibitors either by binding to the recognition sites within the element, or by protein-protein interaction with the transposase. We consider it possible that expression of TclA is similarly accompanied by the generation of a low level of a smaller version of the protein that regulates transposition.

\section{Materials and methods}

Cloning of various plasmids

Generation of the hsp-Tc1A fusion A PCR product containing the C. elegans heat shock promoter-untranslated RNA leader of hsp-16 (position 1018-1398; Stringham et al. 1992) was obtained from template pC31 using primers $2335\left(5^{\prime}\right.$-CCGGCATGCTGGACGGAAATAGTGG) and 2123 (5'-CATTCTTGAAGTTTAGAGAATGAACAG). The Tc1A gene was obtained after PCR using primers 2334 (5'-ATGGAATTCTTAATACTTTGTCGCGTATCC) and $2122\left(5^{\prime}\right.$-CTCTAAACTTCAAGAATGGTAAAATCTGTTGGG), together with template pIM40, which contains a Tc1 element that cosegregates with mutator activity (Mori 1988). As a result of an overlap extension (Horton et al. 1989) between the 5' end of the Tc1A PCR product and the $3^{\prime}$ end of the $h s p$ promoter PCR product, the two fragments could be fused and further amplified by PCR using primers 2334 and $2335\left(25\right.$ cycles; $1 \mathrm{~min}$ at $95^{\circ} \mathrm{C}, 1 \mathrm{~min}$ at $55^{\circ} \mathrm{C}, 1 \mathrm{~min}$ at $72^{\circ} \mathrm{C}$ ). Products were recovered and restricted with $S p h \mathrm{I}$ and EcoRI and ligated into the SphI-EcoRI-restricted plasmid backbone of pPD26.50 (a derivative of pPD21.28; Fire et al. 1990), which resulted in plasmid pRP465.

Construction of pRP470 A similar PCR strategy was used for the removal of the 41-bp intron from the TclA gene from pIM40 to express TclA in E. coli. The first exon was obtained using primers 2336 (5'-AGCCATATGGTAAAATCTGTTGGGTG) and 2407 (5'-CTCAGTTTGGTACTTCTTG), and the second exon was amplified using primers 2409 (5'-CAAGAAGTACCAAACTGAGAAATCCGTCGCTTTGAG) and 964 (5'-ATGAGATCTTAATACTTTGTCGCGTATCC). The fused PCR product was amplified using primers 2336 and 964, recovered and subsequently restricted with $N d e I$ and $B g I I I$, and ligated into the NdeI and BamHI sites of pET3c (Rosenberg et al. 1987) to yield pRP470.

Construction of deletion mutants Carboxy-terminal deletion pN207 resulted from linearization of pRP470 with ApaI, the site of which was made blunt by T4 DNA polymerase in the presence of dNTPs, and subsequent religation of the product, which results in an in-frame stop codon immediately downstream of the disrupted ApaI site. Bal31 deletions were generated from ApaI-linearized pRP470. Reaction products from different time points were digested with PstI and run on an agarose gel. Carboxy-terminal deletion fragments were purified and cloned into pET3c digested with PstI and BamHI (made blunt by Klenow and dNTPs). Deletion endpoints were verified by sequencing the final constructs pN54, pN78, pN101, and pN153 in which the nomenclature corresponds with the number of authentic amino-terminal TclA codons. Finally, pN63 was generated by cloning the PstI-StuI fragment with the $5^{\prime}$ end of the Tc1A gene from pRP470 into pET3c digested with PstI and BamHI. The use 
of a translational stop codon in the vector-derived sequences resulted in the synthesis of a fusion protein with an additional 20 or 23 amino acids at the carboxyl terminus.

\section{Protein expression and purification}

TclA was expressed in E. coli strain BL21 pLysS grown in Luria broth at $30^{\circ} \mathrm{C}$. When the cells reached an O.D. of 0.5 at $600 \mathrm{~nm}$, IPTG was added to a concentration of $0.4 \mathrm{~mm}$ and incubation was continued for $2.5 \mathrm{hr}$. Bacteria were collected by centrifugation and resuspended in $\mathrm{H}$ buffer [ $25 \mathrm{mM}$ HEPES ( $\mathrm{pH} 7.5), 0.1$ mM EDTA, $15 \%$ glycerol, $10 \mathrm{~mm} \mathrm{MgCl}_{2}, 0.1 \% \mathrm{NP}-40,2 \mathrm{~mm}$ DTT] containing $1 \mathrm{mM}$ PMSF and $1 \mathrm{M} \mathrm{NaCl}$. Cell lysis was achieved by sonication, and the insoluble material was removed by centrifugation.

The TclA derivative was purified by the addition of one volume of saturated ammonium sulfate $(\mathrm{pH} 7.5)$ to the supernatant. Precipitated proteins were collected by centrifugation, resuspended in $1 \mathrm{M} \mathrm{NaCl}$, and dialyzed against $200 \mathrm{mM} \mathrm{NaCl}$ in $\mathrm{H}$ buffer. This material was loaded onto a S-Sepharose FF column. The Tc1A-related polypeptide was eluted with a step from 350 to $550 \mathrm{mM} \mathrm{NaCl}$ in $\mathrm{H}$ buffer. This material was dialyzed against $\mathrm{H}$ buffer containing $100 \mathrm{~mm} \mathrm{NaCl}$ and passed through a Q-Sepharose FF (Pharmacia) column. The flowthrough fraction had a total protein concentration of $\sim 1 \mathrm{mg} / \mathrm{ml}$ and contained $\sim 25$ $\mu \mathrm{g} / \mathrm{ml}$ of a $18-\mathrm{kD}$ polypeptide, which reacts with a polyclonal serum raised against TcA.

\section{Generation and analysis of transgenic animals}

A transgenic Bristol (N2) line (NL224) was obtained after microinjection (Mello et al. 1991) of $200 \mu \mathrm{g} / \mathrm{ml}$ of pRF4 (Kramer et al. 1990), together with $5 \mu \mathrm{g} / \mathrm{ml}$ of plasmid pRP465. Transgenic animals were grown at $18^{\circ} \mathrm{C}$. DNA was isolated from C. elegans as described (Sulston and Hodgkin 1988). Tc1 insertions in gpa-2 were detected by two rounds of PCR $(30$ cycles; 1 min at $95^{\circ} \mathrm{C}, 1 \mathrm{~min}$ at $55^{\circ} \mathrm{C}, 1 \mathrm{~min}$ at $\left.72^{\circ} \mathrm{C}\right)$. The first PCR used as gpa-2 primer 3172 (5'-GCAACTGCTCGAAACCTCCGCAAC) and Tc1 primer L1 (5'-CGTGGGTATTCCTTGTTCGAAGCCAGCTACAATGGCTTTCT). The second PCR used $1 \mu l$ of a 100fold dilution of the first PCR as template in combination with gpa-2 primer 3173 (5'-GTGCTAGTTTCAATCCAAGATC) and Tc1 primer L2 (5'-TCAAGTCAAATGGATGCTTGAG).

RNA was isolated as described in Rosenquist and Kimble (1988). Northern analysis was performed as described in Sambrook et al. (1989). A cDNA was generated using a Tc1A genespecific primer (964) and $10 \mu \mathrm{g}$ of total RNA obtained from transgenic animals immediately after a $3-\mathrm{hr}$ heat shock at $33^{\circ} \mathrm{C}$. The cDNA was amplified by PCR using primers 964 and 2336 (50 cycles; $1 \mathrm{~min}$ at $95^{\circ} \mathrm{C}, 1 \mathrm{~min}$ at $55^{\circ} \mathrm{C}, 1 \mathrm{~min}$ at $72^{\circ} \mathrm{C}$ ). The product of an asymmetric PCR (excess 964) was sequenced using primer 2336 .

Preparation of cytoplasmic and nuclear extracts of C. elegans One volume of worms was mixed with two volumes of nuclear isolation buffer [NIB: $0.5 \mathrm{M}$ sucrose, $25 \mathrm{mM}$ HEPES ( $\mathrm{pH} 7.5$ ), 25 $\mathrm{mM} \mathrm{KCl}, 0.1 \mathrm{~mm}$ EDTA, $0.15 \mathrm{~mm}$ spermine, $0.15 \mathrm{~mm}$ spermidine, $10 \mathrm{~mm}$ DTT, $10 \mathrm{~mm} \mathrm{MgCl}_{2}, 0.1 \mathrm{~mm}$ PMSF]. This suspension was ground in liquid nitrogen until a fine white powder was obtained. Then, four volumes of NIB were added, and the cells were broken by 40 strokes in a Dounce homogenizer. Debris was removed by centrifugation at $200 \mathrm{~g}$ for $2 \mathrm{~min}$. Nuclei were collected by centrifugation of the supernatant for $5 \mathrm{~min}$ in an Eppendorf centrifuge. The supernatant contained the cytoplasmic fraction $(\sim 10 \mathrm{mg} / \mathrm{ml}$ of protein). Nuclei were extracted with two volumes of buffer $\mathrm{H}$ containing $500 \mathrm{mM} \mathrm{NaCl}$. After homogenizing in a Dounce, the insoluble material was removed by centrifugation. The protein concentration of the nuclear extract was $\sim 5 \mathrm{mg} / \mathrm{ml}$.

\section{Gel retardation and DNase I footprinting}

Probes and oligonucleotides Double-stranded oligonucleotides corresponding to regions of the ends of Tc1 [5'-AGTGGATATCTTTTTGGCCAGCACTGTA and its complement $(\mathrm{bp}-1$ to +26$)$ or 5'-ACCAAAAGTGGATATCTTTTTGGCCAGC and its complement (bp +5 to +32 )] were subcloned into the polylinker site of pUCl8. The nonspecific doublestranded oligonucleotide (5'-AAACCGAGGCAGGAAAATCCCTAGCAGG and its complement) was also cloned into the polylinker of pUC18. Gel-retardation probes were obtained by end-labeling appropriate restriction fragments with $\left[\alpha^{32} \mathrm{P}\right] \mathrm{dATP}$ and Klenow. A footprint probe was obtained from pRSA, in which the terminal 225 bp of Tc1 was cloned as a BamHI-RsaI fragment from pRP4 into BamHI - SmaI-digested pUC18. Footprint probes were obtained by restriction of pRSA with HindIII and end-labeling either with $\left[\alpha^{32} \mathrm{P}\right] \mathrm{dATP}$ and Klenow or by using T4 polynucleotide kinase and $\left[\gamma^{32} \mathrm{P}\right] \mathrm{ATP}$, followed by subsequent digestion with EcoRI.

TclA-DNA complexes were formed in $20 \mathrm{mM}$ HEPES $/ \mathrm{pH}$ 7.5), $0.1 \mathrm{~mm}$ EDTA, $5 \mathrm{~mm}$ spermidine, $0.1 \mathrm{mg} / \mathrm{ml}$ of BSA, 100$200 \mathrm{mM} \mathrm{NaCl}, 1 \mathrm{~mm} \mathrm{DTT}$, and $0.1 \% \mathrm{NP}-40$ using 5 fmoles of probe in a total volume of $20 \mu \mathrm{l}$. Standard reactions used $2 \mu \mathrm{l}$ of C. elegans extract (together with $1 \mu \mathrm{g}$ of poly[d(I-C)] or $1 \mu \mathrm{l}$ of total $E$. coli extract (together with $2 \mu \mathrm{g}$ of poly[d(I-C)]. After 15 min of incubation on ice, $5 \mu \mathrm{l}$ of $25 \%$ Ficoll was added and the samples were loaded onto a $5 \%$ polyacrylamide gel in TAE buffer (Sambrook et al. 1989).

Footprinting reactions $(20 \mu \mathrm{l})$ contained $200 \mathrm{mM} \mathrm{NaCl}, 1 \mathrm{mM}$ DTT, $0.1 \%$ NP-40, $20 \mathrm{~mm}$ HEPES (pH 7.5), $0.1 \mathrm{mg} / \mathrm{ml}$ of BSA, $0.1 \mathrm{mM}$ EDTA, $2 \mu \mathrm{g}$ of poly[d(I-C)], $100 \mathrm{ng}$ of calf thymus DNA, and $5 \mathrm{fmoles}$ of probe in the absence or presence of $1 \mu \mathrm{l}$ purified 18- $\mathrm{kD} \mathrm{TclA}$ derivative. After $15 \mathrm{~min}$ of incubation at $20^{\circ} \mathrm{C}, 2 \mu \mathrm{l}$ of DNase I $\left(0.01 \mathrm{mg} / \mathrm{ml}\right.$ in $100 \mathrm{mM} \mathrm{CaCl}$ and $\left.100 \mathrm{mM} \mathrm{MgCl}_{2}\right)$ was added. Reactions were stopped after $1 \mathrm{~min}$ by the addition of $2 \mu$ l of stop solution [ $5 \%$ SDS, $250 \mathrm{mM}$ Tris (pH 7.4), $250 \mathrm{~mm}$ EDTA], and the DNA products were ethanol precipitated, resuspended in formamide dye, and separated on a $6 \%$ sequencing gel.

\section{Western and South-Western blots}

Protein samples were subjected to electrophoresis in SDS-polyacrylamide gels, blotted to nitrocellulose, and either probed with a polyclonal serum against TclA (Schukkink and Plasterk 1990 ) or with a DNA probe $[2 \mathrm{ng} / \mathrm{ml}$, linearized vector DNA with no Tc1 similarity labeled by random priming (Feinberg and Vogelstein 1984) using [ $\left.\alpha^{32} \mathrm{P}\right] \mathrm{dATP}$ and Klenow] essentially as described by Krogstad and Champoux (1990).

\section{Acknowledgments}

We thank Drs. Stringham and Candido for pC31, Dr. Fire for pPD26.50, Dr. Mori for pIM40, and Dr. Kramer for pRF4. We thank José Groenen for assistance with the microinjections. Stimulating discussions with our colleagues were greatly appreciated. We thank Piet Borst, Sean Colloms, Kenny Simmen, and Kees Vink for critically reading of the manuscript. This work was supported by a Pioneir grant of the Dutch science foundation NWO to R.H.A.P.

The publication costs of this article were defrayed in part by 
payment of page charges. This article must therefore be hereby marked "advertisement" in accordance with 18 USC section 1734 solely to indicate this fact.

\section{References}

Altschul, S.L., W. Gish, W. Miller, E.W. Myers, and D.J. Lipman. 1990. Basic local alignment search tool. I. Mol. Biol. 215: 403-410.

Brezinsky, L., G.V.L. Wang, T. Humphreys, and J. Hunt. 1990. The transposable element Uhu from Hawaiian Drosophila - Member of the widely dispersed class of Tc1-like elements. Nucleic Acids Res. 18: 2053-2059.

Collins, J., B. Saari, and P. Anderson. 1987. Activation of a transposable element in the germline but not the soma of Caenorhabditis elegans. Nature 328: 726-728.

Collins, J., E. Forbes, and P. Anderson. 1989. The Tc3 family of transposable elements in Ceanorhabditis elegans. Genetics 121: 47-55.

Craigie, R., M. Mizuuchi, and K. Mizuuchi. 1984. Site-specific recognition of the bacteriophage $\mathrm{Mu}$ ends by the $\mathrm{Mu}$ A protein. Cell 39: 387-394.

Derbyshire, K.M. and N.D.F. Grindley. 1992. Binding of the IS903 transposase to its inverted repeat in vitro. EMBO $/$. 11: 3449-3455.

Dombroski, A.J., W.A. Walter, M.T. Record Jr., D.A. Siegele, and C.A. Gross. 1992. Polypeptides containing highly conserved regions of transcription initiation factor $\sigma^{70}$ exhibit specificity of binding to promoter DNA. Cell 70: 501-512.

Emmons, S.W. and L. Yesner. 1984. High-frequency excision of transposable element Tc1 in the nematode Caenorhabditis elegans is limited to somatic cells. Cell 36: 599-605.

Escoubas, J.M., M.F. Prère, O. Fayet, I. Salvignol, D. Galas, D. Zerbib, and M. Chandler. 1991. Translational control of transposition activity of the bacterial insertion sequence IS1. EMBO I. 10: 705-712.

Feinberg, A.P. and B. Vogelstein. 1984. A technique for radiolabeling DNA restriction endonuclease fragments to high specific activity. Anal. Biochem. 137: 266-267.

Fino Silva, I. and R.H.A. Plasterk. 1990. Characterization of a G-protein $\alpha$-subunit gene from the nematode Caenorhabditis elegans. J. Mol. Biol. 215: 483-487.

Fire, A., S. White Harrison, and D. Dixon. 1990. A modular set of lacZ fusion vectors for studying gene expression in C. elegans. Gene 93: 189-198.

Gierl, A., S. Lutticke, and H. Saedler. 1988. TnpA product encoded by the transposable element En-l of Zea mays is a DNA binding protein. EMBO J. 7: 4045-4053.

Harris, L.J., D.L. Baillie, and A.M. Rose. 1988. Sequence identity between an inverted repeat family of transposable elements in Drosophila and Caenorhabditis. Nucleic Acids Res. 16: 5991-5997.

Heierhorst, J., K. Lederis, and D. Richter. 1992. Presence of a member of the Tc1-like transposon family from nematodes and Drosophila within the vasotocin gene of a primitive vertebrate, the Pacific hagfish Eptatretrus stouti. Proc. Natl. Acad. Sci. 89: 6798-6802.

Henikoff, S. 1992. Detection of Caenorhabditis transposon homologs in diverse organisms. New Biol. 4: 382-388.

Henikoff, S. and R.H.A. Plasterk. 1988. Related transposons in C. elegans and D. melanogaster. Nucleic Acids Res. 16: 6234.

Horton, R.M., H.D. Hunt, S.N. Ho, J.K. Pullen, and L.R. Pease. 1989. Engineering hybrid genes without the use of restriction enzymes: Gene splicing by overlap extension. Gene 77:
$61-68$.

Huisman, O., P.E. Errada, L. Signon, and N. Kleckner. 1989. Mutational analysis of IS10's outside end. EMBO J. 8: 21012109.

Hupp, T.A., D.W. Meek, C.A. Midgley, and D.P. Lane. 1992. Regulation of the specific DNA binding function of p53. Cell 71: 875-886.

Isberg, R.R., A.L. Lazaar, and M. Syvanen. 1982. Regulation of Tn 5 by the right-repeat proteins: Control at the level of the transposition reaction? Cell 30: 883-892.

Johnson, R.C., J.C.P. Yin, and W.S. Reznikoff. 1982. Control of Tn 5 transposition in Escherichia coli is mediated by protein from the right repeat. Cell 30: 873-882.

Kaufman P.D., R.F. Doll, and D.C. Rio. 1989. Drosophila P element transposase recognizes internal $P$ element DNA sequences. Cell 59: 359-371.

Kramer, J.M., R.P. French, E.-C. Park, and J.J. Johnson. 1990. The Caenorhabditis elegans rol-6 gene, which interacts with the sqt-1 collagen gene to determine organismal morphology, encodes a collagen. Mol. Cell. Biol. 10: 2081-2089.

Krogstad, P.A. and J.J. Champoux. 1990. Sequence-specific binding of DNA by the Moloney murine leukemia virus integrase protein. J. Virol. 64: 2796-2801.

Kunze, R. and P. Starlinger. 1989. The putative transposase of transposable element Ac from Zea mays L. interacts with subterminal sequences of Ac. EMBO I. 8: 3177-3185.

Lavoie, B.D., B.S. Chan, R.G. Allison, and G. Chaconas. 1991. Structural aspects of a higher order nucleoprotein complex: Induction of an altered DNA structure at the Mu-host junction of the Mu Type 1 transpososome. EMBO /. 10: 30513059.

Mello, C.C., J.M. Kramer, D. Stinchcomb, and V. Ambros. 1991. Efficient gene transfer in C. elegans: Extrachromosomal maintenance and integration of transforming sequences. EMBO /. 10: 3959-3970.

Misra, S. and D.C. Rio. 1990. Cytotype control of Drosophila P element transposition: The $66 \mathrm{kd}$ protein is a repressor of transposase activity. Cell 62: 269-284.

Mizuuchi, M., T.A. Baker, and K. Mizuuchi. 1991. DNase protection analysis of the stable synaptic complexes involved in Mu transposition. Proc. Natl. Acad. Sci. 84: 1809-1813.

Mori, I. 1988. "Analysis of germline transposition and excision of Tcl transposable elements in Caenorhabditis elegans." Ph.D. thesis. Washington University, St. Louis, MO.

Mori, I., G.M. Benian, D.G. Moerman, and R.H. Waterston. 1988a. Transposable element Tc1 of Caenorhabditis elegans recognizes specific target sequences for integration. Proc. Nat1. Acad. Sci. 85: 861-864.

Mori, I., D.G. Moerman, and R.H. Waterston. 1988b. Analysis of a mutator activity necessary for germline transposition and excison of Tc1 transposable elements in Caenorhabditis elegans. Genetics 120: 397-407.

Plasterk, R.H.A. 1991. The origin of footprints of the Tc1 transposon of Caenorhabditis elegans. EMBO J. 10: 1919-1925.

Prasad, S.S., L.J. Harris, D.L. Baillie, and A.M. Rose. 1991. Evolutionarily conserved regions in Caenorhabditis transposable elements deduced by sequence comparison. Genome 34: 6-12.

Rosenberg, A.H., B.N. Lade, D.-s. Chui, S.-W. Lin, J.J. Dunn, and F.W. Studier. 1987. Vectors for selective expression of cloned DNAs by T7 RNA polymerase. Gene 56: 125-135.

Rosenquist, T.A. and J. Kimble. 1988. Molecular cloning and transcript analysis of fem-3, a sex-determination gene in Caenorhabditis elegans. Genes \& Dev. 2: 606-616.

Rosenzweig, B., L.W. Liao, and D. Hirsch. 1983a. Sequence of the C. elegans transposable element Tcl. Nucleic Acids Res. 
12: 4201-4209.

Rosenzweig, B., L.W. Liao, and D. Hirsch. 1983b. Target sequences for the $\mathrm{C}$. elegans transposable element Tc1. $\mathrm{Nu}$ cleic Acids Res. 11: 7137-7140.

Sambrook, J., E.F. Fritsch, and T. Maniatis. 1989. Molecular cloning: A laboratory manual. Cold Spring Harbor Laboratory Press, Cold Spring Harbor, New York.

Schukkink, R.F. and R.H.A. Plasterk. 1990. TcA, the putative transposase of the C. elegans Tcl transposon, has an N-terminal DNA binding domain. Nucleic Acids Res. 18: 895900.

Stalder, R., P. Caspers, F. Olasz, and A. Werner. 1990. The N-terminal domain of the insertion sequence 30 transposase interacts specifically with the terminal inverted repeats of the element. J. Biol. Chem. 265: 3757-3762.

Stringham, E.G., D.K. Dixon, D. Jones, and E.P.M. Candido. 1992. Temporal and spatial expression patterns of the small heat shock (hsp16) genes in transgenic Caenorhabditis elegans. Mol. Biol. Cell 3: 221-233.

Sulston, J.E. and J. Hogdkin. 1988. Methods. In The nematode Caenorhabditis elegans (ed. W.B. Wood), pp. 587-606. Cold Spring Harbor Laboratory, Cold Spring Harbor, New York. 


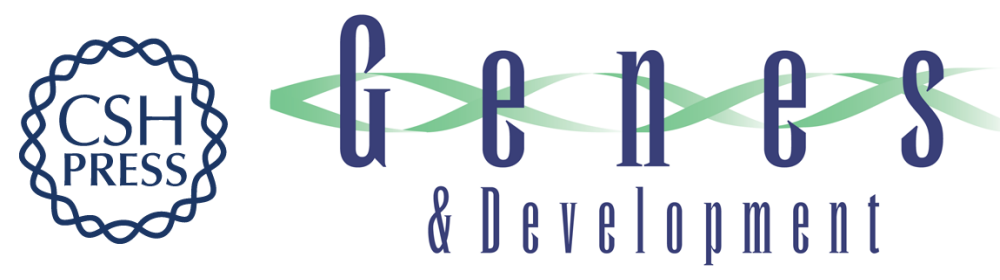

\section{Characterization of the Caenorhabditis elegans Tc1 transposase in vivo and in vitro.}

J C Vos, H G van Luenen and R H Plasterk

Genes Dev. 1993, 7:

Access the most recent version at doi:10.1101/gad.7.7a.1244

References This article cites 41 articles, 9 of which can be accessed free at: http://genesdev.cshlp.org/content/7/7a/1244.full.html\#ref-list-1

License

Email Alerting

Service

Receive free email alerts when new articles cite this article - sign up in the box at the top right corner of the article or click here.

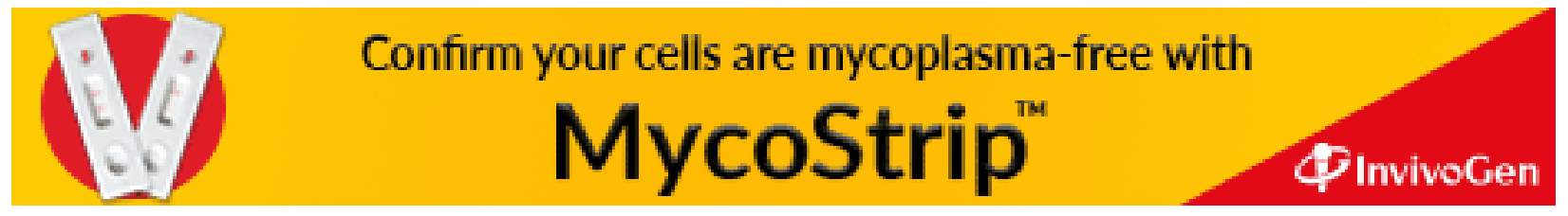

